\title{
Anisotropic cosmological models with perfect fluid and dark energy revisited
}

\author{
Bijan Saha \\ Laboratory of Information Technologies \\ Joint Institute for Nuclear Research, Dubna \\ 141980 Dubna, Moscow region, Russid*
}

(Dated: November 6, 2018)

\begin{abstract}
We consider a self-consistent system of Bianchi type-I (BI) gravitational field and a binary mixture of perfect fluid and dark energy. The perfect fluid is taken to be the one obeying the usual equation of state, i.e., $p=\zeta \varepsilon$, with $\zeta \in[0,1]$ whereas, the dark energy is considered to be obeying a quintessence-like equation of state. Exact solutions to the corresponding Einstein equations are obtained. The model in consideration gives rise to a Universe which is spatially finite. Depending on the choice of problem parameters the Universe is either close with a space-time singularity, or an open one which is oscillatory, regular and infinite in time.

PACS numbers: 04.20.Ha, 03.65.Pm, 04.20.Jb
\end{abstract}

Keywords: Bianchi type I (BI) model, perfect fluid, dark energy

\section{INTRODUCTION}

The discovery that the expansion of the Universe is accelerating [1] has promoted the search for new types of matter that can behave like a cosmological constant [2, 3] by combining positive energy density and negative pressure. This type of matter is often called quintessence. Zlatev et al. [4] showed that "tracker field", a form of qiuntessence, may explain the coincidence, adding new motivation for the quintessence scenario.

An alternative model for the dark energy density was used by Kamenshchik et al. [5], where the authors suggested the use of some perfect fluid but obeying "exotic" equation of state. This type of matter is known as Chaplygin gas. In doing so the authors considered mainly a spatially flat, homogeneous and isotropic Universe described by a Friedmann-Robertson-Walker (FRW) metric.

The theoretical arguments and recent experimental data, which support the existence of an anisotropic phase that approaches an isotropic one, lead to consider the models of Universe with anisotropic back-ground. Since the modern-day Universe is almost isotropic at large, its simplicity and evolution into a FRW Universe makes the BI Universe a prime candidate for studying the possible effects of an anisotropy in the early Universe on present-day observations. In a number of papers, e.g., [6, 7], we have studied the role of a nonlinear spinor and/or a scalar fields in the formation of an anisotropic Universe free from initial singularity. It was shown that for a suitable choice of nonlinearity and the sign of $\Lambda$ term the model in question allows regular solutions and the Universe becomes isotropic in the process of evolution. Recently Khalatnikov et al. [8] studied the Einstein equations for a BI Universe in the presence of dust, stiff matter and cosmological constant. In a recent paper [9] the author studied a self-consistent system of Bianchi type-I (BI) gravitational

*Electronic address: saha@ @thsun1.jinr.ru, bijan@jinr.ru, URL: http://thsun1.jinr.ru/〜saha/ 
field and a binary mixture of perfect fluid and dark energy given by a cosmological constant. The perfect fluid in that paper was chosen to be the one obeying either the usual equation of state, i.e., $p=\zeta \varepsilon$, with $\zeta \in[0,1]$ or a van der Waals equation of state. That paper was followed by another where we studied the evolution of an initially anisotropic Universe given by a BI spacetime and a bimnary mixture of a perfect fluid obeying the equation of state $p=\zeta \varepsilon$ and a dark energy given by either a quintessence or a Chaplygin gas [10]. It should be mentioned that the inclusion of dark energy does not eliminate initial singularity of the model and the space-time in those cases is ever-expanding. In order to obtain a singularity-free Universe in the present paper we introduce a modified version of quintessence-like dark energy, which at the same time is able to explain the accelerated expansion.

\section{BASIC EQUATIONS}

The gravitational field in our case is given by a Bianchi type I (BI) metric in the form

$$
d s^{2}=d t^{2}-a^{2} d x^{2}-b^{2} d y^{2}-c^{2} d z^{2},
$$

with the metric functions $a, b, c$ being the functions of time $t$ only.

The Einstein field equations for the BI space-time we write in the form

$$
\begin{aligned}
\frac{\ddot{b}}{b}+\frac{\ddot{c}}{c}+\frac{\dot{b}}{b} \frac{\dot{c}}{c} & =\kappa T_{1}^{1}, \\
\frac{\ddot{c}}{c}+\frac{\ddot{a}}{a}+\frac{\dot{c}}{c} \frac{\dot{a}}{a} & =\kappa T_{2}^{2}, \\
\frac{\ddot{a}}{a}+\frac{\ddot{b}}{b}+\frac{\dot{a} \frac{\dot{b}}{a}}{b} & =\kappa T_{3}^{3}, \\
\frac{\dot{a}}{a} \frac{\dot{b}}{b}+\frac{\dot{b}}{b} \frac{\dot{c}}{c}+\frac{\dot{c}}{c} \frac{\dot{a}}{a} & =\kappa T_{0}^{0} .
\end{aligned}
$$

Here $\kappa$ is the Einstein gravitational constant and over-dot means differentiation with respect to $t$. The energy-momentum tensor of the source is given by

$$
T_{\mu}^{v}=(\varepsilon+p) u_{\mu} u^{v}-p \delta_{\mu}^{v},
$$

where $u^{\mu}$ is the flow vector satisfying

$$
g_{\mu \nu} u^{\mu} u^{v}=1 .
$$

Here $\varepsilon$ is the total energy density of a perfect fluid and/or dark energy density, while $p$ is the corresponding pressure. $p$ and $\varepsilon$ are related by an equation of state which will be studied below in detail. In a co-moving system of coordinates from (2.3) one finds

$$
T_{0}^{0}=\varepsilon, \quad T_{1}^{1}=T_{2}^{2}=T_{3}^{3}=-p .
$$

In view of (2.5) from (2.2) one immediately obtains [6]

$$
\begin{aligned}
& a(t)=D_{1} \tau^{1 / 3} \exp \left[X_{1} \int \frac{d t}{\tau(t)}\right], \\
& b(t)=D_{2} \tau^{1 / 3} \exp \left[X_{2} \int \frac{d t}{\tau(t)}\right], \\
& c(t)=D_{3} \tau^{1 / 3} \exp \left[X_{3} \int \frac{d t}{\tau(t)}\right] .
\end{aligned}
$$


Here $D_{i}$ and $X_{i}$ are some arbitrary constants obeying

$$
D_{1} D_{2} D_{3}=1, \quad X_{1}+X_{2}+X_{3}=0
$$

and $\tau$ is a function of $t$ defined to be

$$
\tau=a b c .
$$

Summation of (2.2a), (2.2b), (2.2c) and 3 times (2.2d) gives the equation for $\tau$ :

$$
\frac{\ddot{\tau}}{\tau}=\frac{3 \kappa}{2}(\varepsilon-p)
$$

whereas, from the conservation law for the energy-momentum tensor for $\varepsilon$ we find

$$
\dot{\varepsilon}=-\frac{\dot{\tau}}{\tau}(\varepsilon+p)
$$

After a little manipulations from (2.8) and (2.9) we find

$$
\dot{\tau}= \pm \sqrt{C_{1}+3 \kappa \varepsilon \tau^{2}}
$$

with $C_{1}$ being an integration constant. Eq. (2.9) can be rewritten in the form

$$
\frac{\dot{\varepsilon}}{(\varepsilon+p)}=-\frac{\dot{\tau}}{\tau}
$$

Taking into account that the pressure and the energy density obey a equation of state of type $p=f(\varepsilon)$, we conclude that $\varepsilon$ and $p$, hence the right hand side of the Eq. (2.8) is a function of $\tau$ only, i.e.,

$$
\ddot{\tau}=\frac{3 \kappa}{2}(\varepsilon-p) \tau \equiv \mathscr{F}(\tau) .
$$

From the mechanical point of view Eq. (2.12) can be interpreted as an equation of motion of a single particle with unit mass under the force $\mathscr{F}(\tau)$. Then the following first integral exists [11]:

$$
\dot{\tau}=\sqrt{2[\mathscr{E}-\mathscr{U}(\tau)]}
$$

Here $\mathscr{E}$ can be viewed as energy and $\mathscr{U}(\tau)$ is the potential of the force $\mathscr{F}$. Comparing the Eqs. (2.10) and (2.13) one finds $\mathscr{E}=C_{1} / 2$ and

$$
\mathscr{U}(\tau)=-\frac{3}{2} \kappa \varepsilon \tau^{2}
$$

Finally, rearranging (2.10), we write the solution to the Eq. (2.8) in quadrature:

$$
\int \frac{d \tau}{\sqrt{C_{1}+3 \kappa \varepsilon \tau^{2}}}=t+t_{0}
$$

where the integration constant $t_{0}$ can be taken to be zero, since it only gives a shift in time.

In what follows we study the Eqs. (2.8) and (2.9) for perfect fluid and/or dark energy for different equations of state obeyed by the source fields. 


\section{UNIVERSE AS A BINARY MIXTURE OF PERFECT FLUID AND DARK ENERGY}

In this section we thoroughly study the evolution of the BI Universe filled with perfect fluid and dark energy in details. Taking into account that the energy density $(\varepsilon)$ and pressure $(p)$ in this case comprise those of perfect fluid and dark energy, i.e.,

$$
\varepsilon=\varepsilon_{\mathrm{pf}}+\varepsilon_{\mathrm{DE}}, \quad p=p_{\mathrm{pf}}+p_{\mathrm{DE}}
$$

the energy momentum tensor can be decomposed as

$$
T_{\mu}^{v}=\left(\varepsilon_{\mathrm{DE}}+\varepsilon_{\mathrm{pf}}+p_{\mathrm{DE}}+p_{\mathrm{pf}}\right) u_{\mu} u^{v}-\left(p_{\mathrm{DE}}+p_{\mathrm{pf}}\right) \delta_{\mu}^{v}
$$

In the above equation $\varepsilon_{\mathrm{DE}}$ is the dark energy density, $p_{\mathrm{DE}}$ its pressure. We also use the notations $\varepsilon_{\mathrm{pf}}$ and $p_{\mathrm{pf}}$ to denote the energy density and the pressure of the perfect fluid, respectively.

In a comoving frame the conservation law of the energy momentum tensor leads to the balance equation for the energy density

$$
\dot{\varepsilon}_{\mathrm{DE}}+\dot{\varepsilon}_{\mathrm{pf}}=-\frac{\dot{\tau}}{\tau}\left(\varepsilon_{\mathrm{DE}}+\varepsilon_{\mathrm{pf}}+p_{\mathrm{DE}}+p_{\mathrm{pf}}\right) .
$$

The dark energy is supposed to interact with itself only and it is minimally coupled to the gravitational field. As a result the evolution equation for the energy density decouples from that of the perfect fluid, and from Eq. (3.2) we obtain two balance equations

$$
\begin{aligned}
\dot{\varepsilon}_{\mathrm{DE}}+\frac{\dot{\tau}}{\tau}\left(\varepsilon_{\mathrm{DE}}+p_{\mathrm{DE}}\right) & =0, \\
\dot{\varepsilon}_{\mathrm{pf}}+\frac{\dot{\tau}}{\tau}\left(\varepsilon_{\mathrm{pf}}+p_{\mathrm{pf}}\right) & =0 .
\end{aligned}
$$

\section{A. Equations of state}

In order to complete the system of equations we need to specify two equations of state for $p_{\mathrm{pf}}$ and $p_{\mathrm{DE}}$.

\section{1. $\quad$ perfect fluid}

There are some equation of states that are commonly used that, although not widely applicable, are obtained as a result of approximate estimates for particular fluid. The barotropic equation of state

$$
p_{\mathrm{pf}}=\zeta \varepsilon_{\mathrm{pf}}
$$

is often assumed. Here $\zeta$ is a constant and lies in the interval $\zeta \in[0,1]$. Note that $0 \leq \zeta \leq 1$ is necessary for the existence of local mechanical stability and for the speed of sound in the fluid to be no greater than the speed of light. Depending on its numerical value, $\zeta$ describes the following types of Universes [12]

$$
\begin{aligned}
& \zeta=0, \quad \text { (dust Universe), } \\
& \zeta=1 / 3, \quad \text { (radiation Universe), } \\
& \zeta \in(1 / 3,1), \quad \text { (hard Universes), } \\
& \zeta=1, \quad\left(\text { Zel }^{\prime} \text { dovich Universe or stiff matter }\right) \text {. }
\end{aligned}
$$


In view of the Eq. (3.4) from (3.3b) one easily finds laws of change of energy density and pressure of a perfect fluid with the expansion of the Universe :

$$
\varepsilon_{\mathrm{pf}}=\varepsilon_{0} / \tau^{(1+\zeta)}, \quad p_{\mathrm{pf}}=\varepsilon_{0} \zeta / \tau^{(1+\zeta)},
$$

where $\varepsilon_{0}$ is the integration constants. In absence of the dark energy one immediately finds

$$
\tau=C t^{2 /(1+\zeta)}
$$

with $C$ being some integration constant. As one sees from (2.6), in absence of a dark energy, for $\zeta<1$ the initially anisotropic Universe eventually evolves into an isotropic FRW one, whereas, for $\zeta=1$, i.e., in case of stiff matter the isotropization does not take place.

\section{Dark energy}

It was mentioned earlier that the dark energy can be given by a $\Lambda$ term, a quintessence or a Chaplygin gas. It was also mentioned that the quintessence was constructed by combining positive energy density and negative pressure and obeys the equation of state

$$
p_{\mathrm{q}}=w_{\mathrm{q}} \varepsilon_{\mathrm{q}}
$$

where the constant $w_{\mathrm{q}}$ varies between -1 and zero, i.e., $w_{\mathrm{q}} \in[-1,0]$.

Here we introduce a modified model of quintessence when the dark energy and the corresponding pressure obeys the following equation of state:

$$
p_{\mathrm{DE}}=-w\left(\varepsilon_{\mathrm{DE}}-\varepsilon_{\mathrm{cr}}\right),
$$

where the constant $w \in[0,1)$. Here $\varepsilon_{\mathrm{cr}}$ some critical energy density. Setting $\varepsilon_{\mathrm{cr}}=0$ one obtains ordinary quintessence. It is well known that as the Universe expands the (dark) energy density decreases. As a result, being a linear negative function of energy density, the corresponding pressure begins to increase. In case of an ordinary quintessence the pressure is always negative, but for a modified quintessence as soon as $\varepsilon_{\mathrm{q}}$ becomes less than the critical one, the pressure becomes positive. In Fig. 1 we illustrate the evolution of pressure corresponding to ordinary and modified quintessence with the expansion of the Universe.

In account of (3.9) from (3.3a) one finds the following relation between $\varepsilon_{\mathrm{DE}}$ and $\tau$ :

$$
\varepsilon_{\mathrm{DE}}=\frac{1}{1-w}\left[\frac{\varepsilon_{1}}{\tau^{1-w}}-w \varepsilon_{\mathrm{cr}}\right]
$$

with $\varepsilon_{1}$ being some integration constant.

\section{B. Exact and numerical solutions}

As soon as the right hand side of the Eq. (2.12) is defined, we can study this equation in details. First we write the solution to the equation in question in quadrature which will be followed by numerical results.

Inserting $\varepsilon_{\mathrm{pf}}$ and $\varepsilon_{\mathrm{q}}$ into 2.15 one now finds

$$
\int \frac{d \tau}{\sqrt{C_{1}+3 \kappa\left(\varepsilon_{0} \tau^{(1-\zeta)}+\left[\varepsilon_{1} /(1-w)\right] \tau^{(1+w)}-\left[w \varepsilon_{\mathrm{cr}} /(1-w)\right] \tau^{2}\right)}}=t+t_{0}
$$




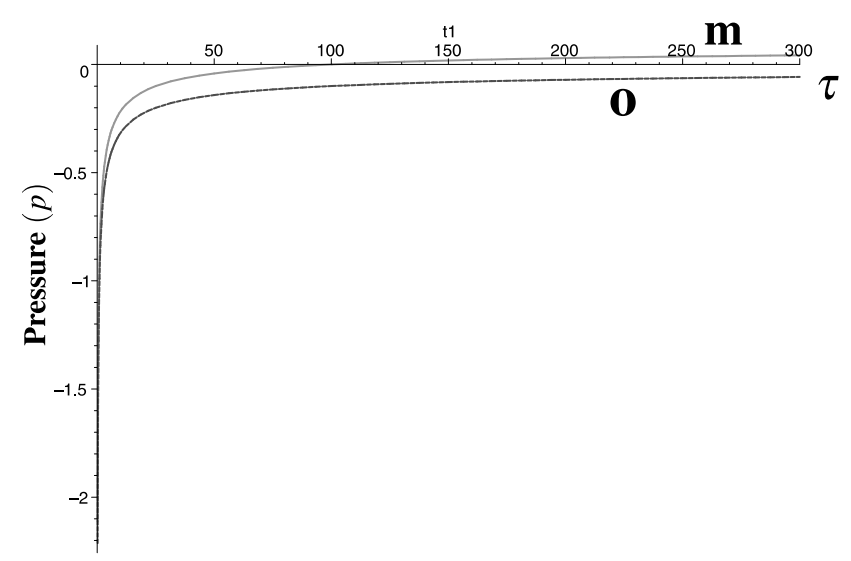

FIG. 1: Evolution of the pressure with the expansion of the BI Universe when it is filled with perfect fluid and dark energy obeying ordinary and modified quintessence given by (3.8) and (3.9), respectively. Here the letters "o" and "m" stand for ordinary and modified, respectively.

Here $t_{0}$ is a constant of integration that can be taken to be trivial. As one sees, the positivity of the radical imposes some restriction on the maximum value of $\tau$, i.e., in this case the model allows oscillatory mode of expansion. It means the dark energy initially acts as a repulsive force resulting in accelerated expansion of the Universe. But as soon as $\varepsilon_{\mathrm{DE}}$ becomes less than $\varepsilon_{\mathrm{cr}}$ the corresponding pressure changes its direction and as a result the Universe begins to contract. In order to give a complete picture, beside the system with a modified quintessence we study the system with an ordinary one as well. It should be noted that the critical energy density $\varepsilon_{\mathrm{DE}}$ should be very small. Here for simplicity we set $\varepsilon_{\mathrm{cr}}=0.01$.

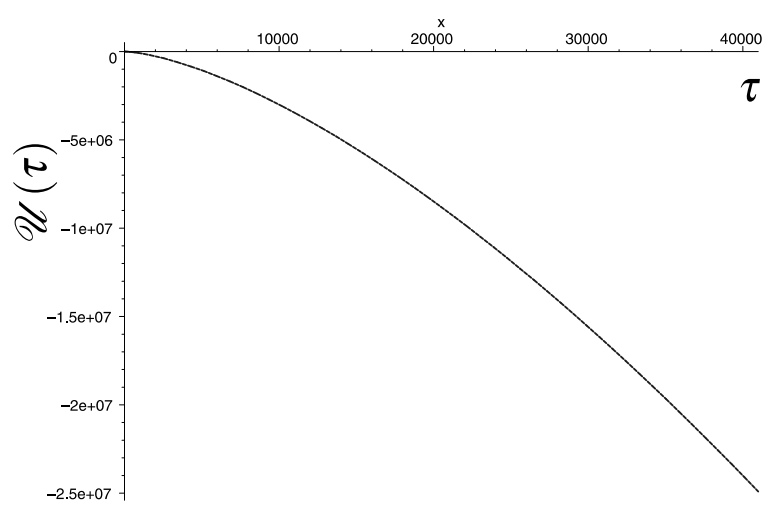

FIG. 2: View of the potential when the BI Universe is filled with a binary mixture of perfect fluid and a quintessence given by 3.8 .

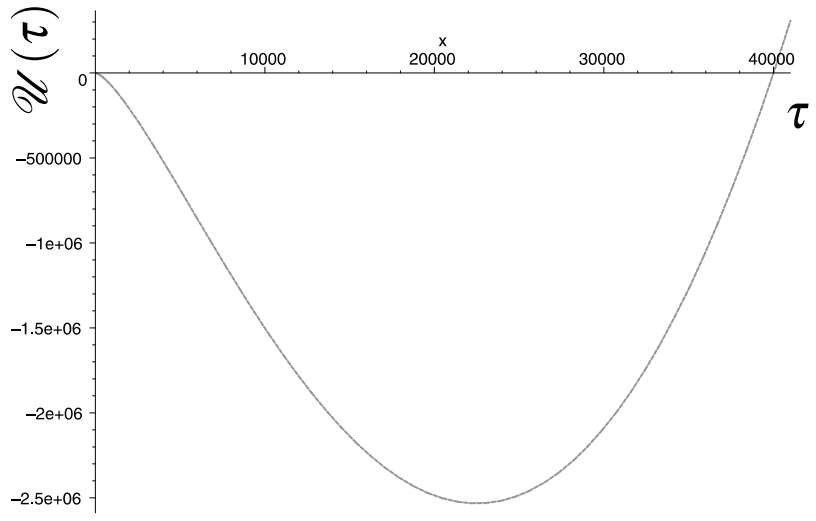

FIG. 3: View of the potential when the BI Universe is filled with a binary mixture of perfect fluid and a modified quintessence-like dark energy given by (3.9).

In Figs. 2 and 3 potentials corresponding to an ordinary and modified quintessence are given. As one sees, the usual quintessence does not allow oscillatory mode of expansion, Universe in this case expands endlessly. A corresponding expansion of the Universe is given in Fig. 4. It should be emphasized that the initially anisotropic Universe in this case evolves into an isotropic FRW one. 
As oppose to the ordinary quintessence a modified quintessence imposes some restriction on the maximum value of $\tau$. As a result the Universe initially expands, but after reaching some maximum it begins to contract. Depending on the choice of the constant, which can be viewed as an energy level, it either shrinks into a point [cf. Fig. 5] thus giving rise to space-time singularity, or begins to expand again after reaching some non-zero minimum, i.e., the Universe in this case experiences the oscillatory mode of expansion [cf. Fig. 6]. Note that to each $\mathscr{E}$ corresponds a particular pair $\left(\tau_{\min }, \tau_{\max }\right)$.

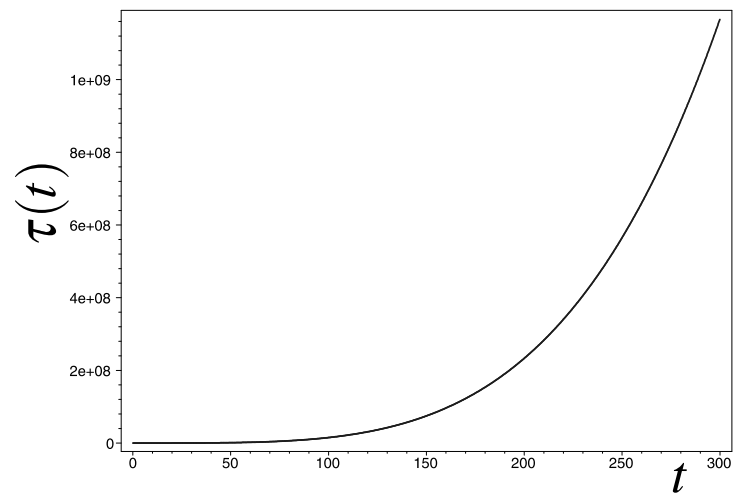

FIG. 4: Evolution of the BI Universe is filled with a binary mixture of perfect fluid and a quintessence. For simplicity, as a perfect fluid we consider only radiation.

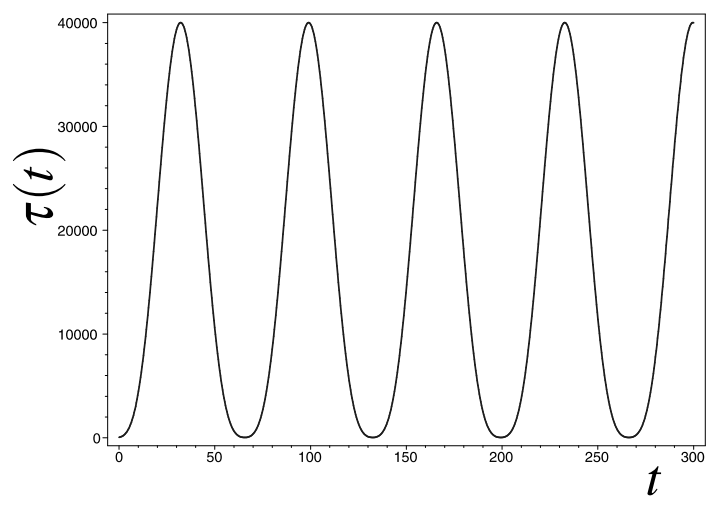

FIG. 6: For $\mathscr{E}<0$ BI Universe filled with a binary mixture of perfect fluid and quintessencelike dark energy admits oscillatory mode of expansion. Here we set $\mathscr{E}=-1000$ with $\tau_{0}=50$.

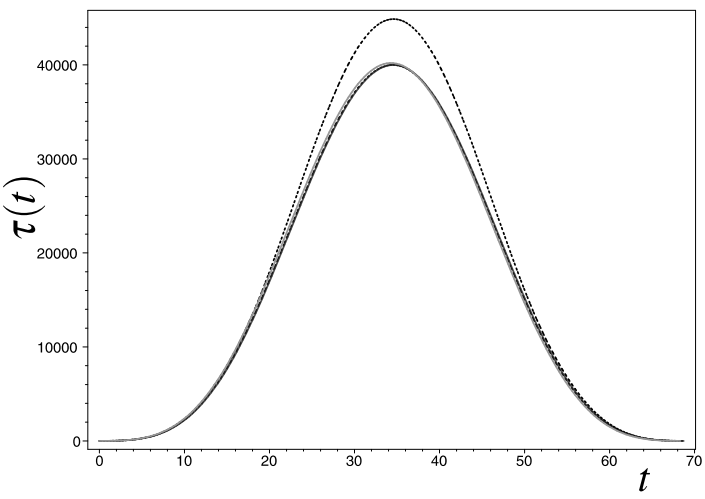

FIG. 5: Evolution of the BI Universe with a modified quintessence. Choosing $\mathscr{E} \geq 0$ (here we set $\mathscr{E}=1$ one obtains nonperiodic picture of evolution. As one sees, different choice of $\zeta$ gives rise to different amplitude, but the overall character of the solution remains unaltered.

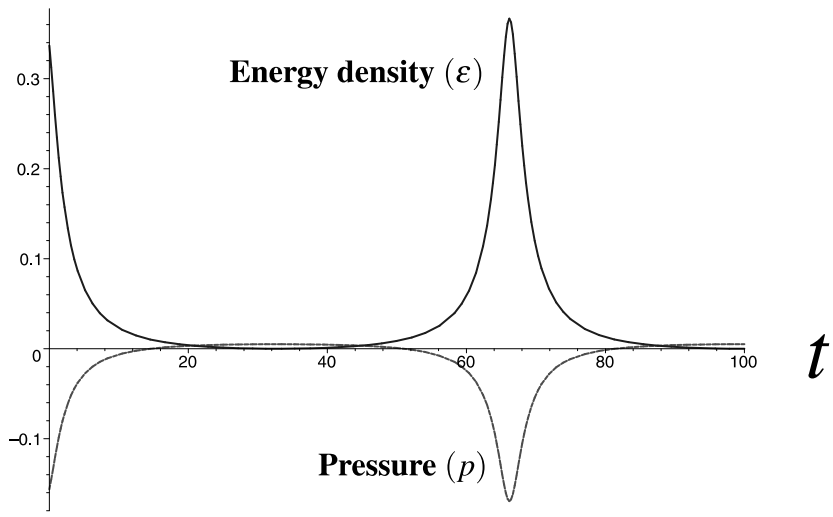

FIG. 7: View of energy density and pressure when BI Universe experiences oscillation as in Fig. 6 
In Fig. 7 we illustrate the evolution of energy density and pressure when the Universe is filled with a binary mixture of perfect fluid given by a radiation and a modified quintessence. As one sees, the initial density is large enough and the pressure is initially negative, with the energy density being less than $\varepsilon_{\mathrm{cr}}$, pressure becomes positive and the Universe begins to contract. As a result the energy density begins to increase and pressure again becomes negative. This results in the oscillatory mode of expansion.

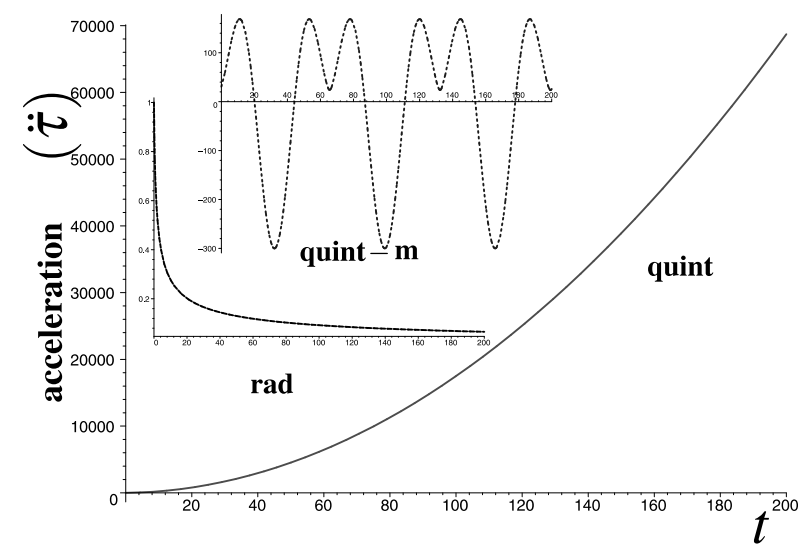

FIG. 8: View of the acceleration for different source fields. Here "rad", "quint" and "quint-m" stand for radiation, a mixture of radiation and an ordinary quintessence and a mixture of radiation and modified quintessence, respectively.

Finally in Fig. 8 we plot the graphic of acceleration verses time. As one sees, if the Universe is filled with perfect fluid only, the process of evolution decelerates with time, while the inclusion of an ordinary quintessence results in an accelerated evolution. If the dark energy is given by a modified quintessence the accelerated expansion is followed by a decelerated one.

\section{CONCLUSION}

A self-consistent system of BI gravitational field filled with a perfect fluid and a dark energy given by a modified version of quintessence has been considered. The exact solutions to the corresponding field equations are obtained. The inclusion of the dark energy into the system gives rise to an accelerated expansion of the model. As a result the initial anisotropy of the model quickly dies away. The modification of the quintessence results in appearing some upper bound of the volume scale, i.e., in this case the Universe is spatially finite. Depending on the choice of the integration constant $\mathscr{E}$, which can be viewed as an energy level, the Universe is either close with a space-time singularity, or an open one which is oscillatory, regular and infinite in time.

[1] N.A. Bachall, J.P. Ostriker, S. Perlmutter, P.J. Steinhardt, Science 284, 1481 (1999).

[2] R.R. Cladwell, R. Dave, and P.J. Steinhardt, Phys. Rev. Lett. 80, 1582 (1998); I. Zlatev, L. Wang, and P.J. Steinhardt, Phys. Rev. Lett. 82, 896 (1999).

[3] V. Sahni and A.A. Starobinsky, Int. J. Mod. Phys. D 9, 373 (2000).

[4] I. Zlatev, L. Wang, and P.J. Steinhardt, Phys. Rev. Lett. 85, 896 (1999). 
[5] A.Yu. Kamenshchik, U. Moschella, and V. Pasquier, Phys. Lett. B. 511, 265 (2001)

[6] Bijan Saha, Phys. Rev. D 64, 123501 (2001) [arXiv: gr-qc/0107013].

[7] Bijan Saha and Todor Boyadjiev, Phys. Rev. D 69, 124010 (2004) [arXiv: gr-qc/0311045].

[8] I.M. Khalatnikov and A.Yu. kamenshchik, Phys. Lett. B 553, 119 (2003).

[9] Saha, Bijan: Anisotropic cosmological models with a perfect fluid and a $\Lambda$ term [arXiv: gr-qc/0411080].

[10] Saha, Bijan: Anisotropic cosmological models with perfect fluid and dark energy [arXiv: gr-qc/0412078].

[11] L.D. Landau and E.M. Lifshitz, Mechanics, Course of Theoretical Physics No. 1 (ButterworthHeinemann, Oxford, 1976).

[12] K.C. Jacobs, Astrophysical Journal 153, 661 (1968). 